\title{
Investigation of physical parameters in ion-beam-heated converters
}

\author{
Abbas Ghasemizad ${ }^{1} \cdot$ Mahboubeh Masoumi $^{1} \cdot$ Leila Gholamzadeh $^{2}$
}

Received: 15 August 2015/Accepted: 18 August 2015/Published online: 10 September 2015

(C) The Author(s) 2015. This article is published with open access at Springerlink.com

\begin{abstract}
The conversion of ion beam energy into thermal X-ray radiation by means of stretched cylindrical volumes is discussed. Converting the kinetic energy of heavy ion beam into radiation energy at high efficiency is important for heavy ion fusion. The conversion efficiency between different materials, low- $\mathrm{Z}$ and high- $\mathrm{Z}$ material, is compared and simulations have been performed by SRIM code. Our results show high-z materials are superior converters. It is found to achieve a high conversion efficiency, a deposition power higher than $10^{16} \mathrm{~W} / \mathrm{cm}^{2}$ is required.
\end{abstract}

Keywords Indirect drive - Heavy ion beam - Converter . X-ray · SRIM code

\section{Introduction}

Inertial confinement fusion (ICF) is a very attractive option for achieving unlimited, safe, and clean source of energy [1]. Heavy ion accelerator is a very promising choice as a reactor driver for inertial confinement fusion because of its high efficiency, excellent repetition rate, final focus and chamber solutions [2,3]. In fuel target implosion, ICF has two means of implosion, namely, direct-driven scheme and indirect-driven scheme [4]. In the former scheme the driver energy is directly deposited

Abbas Ghasemizad

ghasemi@guilan.ac.ir

1 Physics Department, Faculty of Science, Guilan University, Rasht, Iran

2 Physics Department, Yazd University, Yazd, Iran onto an outer layer of the target. In the latter scheme, the beam energy is first converted into soft X-ray which is then used to implode the fusion pellet [5, 6]. Indirect drive has relatively low intrinsic coupling efficiency (ratio of fuel kinetic energy to beam energy) because of the energy penalty in increasing the temperature of the hohlraum walls, but indirect drive targets have intrinsically high uniformity in capsule illumination and are of practical importance [3].The current baseline targets for heavy ion beam drivers are two-sided indirect drives. As shown in Fig. 1 [7], these targets stop the ions in converters distributed within the hohlraum to achieve symmetry. The converter material is strongly heated and it emits thermal radiation into the hohlraum. This thermal radiation is absorbed and re-emitted a number of times at the inner hohlraum surface that leads to a homogeneous radiation field in the cavity [5, 8]. In an analytical model, we have considered a cylindrical converter that irradiated on axis by parallel ion beam. The main aim of this investigation is increasing X-ray flux from converter. There are two main processes where energy is lost in the hohlraum target: (1) the conversion of beam energy into X-Ray in the converters, and (2) the use of this X-ray radiation as driving energy for the implosion [9]. We compare conversion efficiency between different materials and simulations have been performed by SRIM code.

\section{Heavy ion beam interactions with converter}

Heavy ion beams-solid interactions can be used to generate thermal X-rays with properties suitable for utilization in indirect drive Inertial Confinement Fusion [10]. The main differences in energy deposition between ion beams and lasers are that the ions penetrate and deposit their energy 


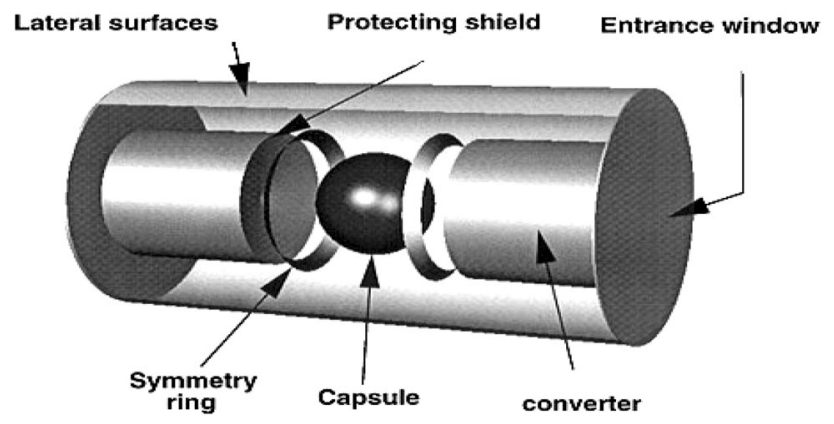

Fig. 1 Typical hohlraum configuration

well inside the target. There is no critical plasma density. Preheating radiation such as suprathermal electrons or hard $\mathrm{X}$-rays and the plasma effects (turbulence, suprathermal electron generation, thermal conduction inhibition) do not arise in ion beam driven. The ions are stopped at a welldefined distance. The ion beams cannot reflect from the target, and the absorption efficiency is $100 \%$. Most of their energy is released near the end of the ion range and very little before. This phenomenon is known as the Bragg peak $[11,12]$.

\section{Converter model}

Converting the kinetic energy of heavy ion beams into radiation energy at high efficiency is important for heavy ion fusion. Indirectly driven high gain ICF pellets depend on high conversion efficiency (ratio of the total radiated energy to the absorbed beam energy) [13]. The energy of an ion beam hitting a target is the sum of internal energy $E_{\text {int }}$ and kinetic energy $E_{\text {kin }}$ of the target material, and radiation energy $E_{\text {rad }}$ as follows:

$E_{\mathrm{b}}=E_{\text {int }}+E_{\text {kin }}+E_{\text {rad }}$

According to the main constraints given by Heavy Ion Driven Inertial Fusion (HIDIF) study, the energy of the ions beam should be greater than $10 \mathrm{GeV}$ per nucleus of $A \approx 200$ [7].

For a given target configuration, total mass of the converter material is:

$M_{\text {con }}=\pi r_{0}^{2} R N_{\text {con }}$

which is to be heated to its working temperature of $T_{r}=250-300 \mathrm{eV}$; here $N_{\text {con }}$ is the number of X-Rays converters, $R\left[\mathrm{~g} / \mathrm{cm}^{2}\right]$ is the range of the beam ions and $r_{0}$ is the initial radius of converter that it is equal to the radius of the focused beam. The parameter $M_{\text {con }}$ is a critical parameter because it determines the energy required to heat the converter, $E_{\text {int }}$. The energy spent to heat up the converter mass constitutes one of the major loss terms in the energy balance of the indirect drive targets:

$E_{\text {int }}=M_{\text {con }} e(T)$

The specific internal energy $e(T)$ is $20-30 \mathrm{MJ} / \mathrm{g}$ at 250 $300 \mathrm{eV}$ temperature, depending on the material. It is approximated by the power law expression $e(T)=e_{0} T^{\mu}$. The parameters $e_{0}$ and $\mu$ depend on the material. The heated converter is assumed to be optically thick; it expands and radiates at its surface during a time interval of $t_{r}=t_{\mathrm{b}}-t_{\mathrm{h}}$, where the total beam time $t_{\mathrm{b}}=\frac{r_{0}}{c_{\mathrm{s}}}$ is set equal to the disintegration time of the converter, $t_{\mathrm{h}}=\frac{e(T)}{P}$ is the time required for heating the converter to the working temperature, and $E_{\mathrm{b}}=M P t_{\mathrm{b}}$, where $P$ is the specific deposition power and also $c_{s}=\sqrt{(\gamma-1) e(T)}$ is the isothermal sound velocity. The kinetic energy and the radiated energy are given by:

$E_{\text {kin }}=\rho_{0} c_{\mathrm{s}}^{3} F t_{r}$

$E_{\mathrm{rad}}=\sigma T^{4} \mathrm{~F} t_{r}$

Also power balance of converter operation requires:

$M_{\text {con }} P=\left(\rho_{0} c_{\mathrm{s}}^{3}+\sigma_{B} T^{4}\right) F$

Taking the stretched cylindrical configuration, one finds $\frac{M}{F} \approx \frac{\rho_{0} r_{0}}{2}$ and $F$ is the surface area of the converter. One can rewrite Eq. 6 in the form:

$\frac{P}{P_{\mathrm{cr}}}=\left[\left(\frac{T}{T_{\mathrm{cr}}}\right)^{3 \mu / 2}+\left(\frac{T}{T_{\mathrm{cr}}}\right)^{4}\right] / 2$

where the critical temperature is:

$T_{\text {cr }}=\left[\left((\gamma-1) e_{0}\right)^{3 / 2} \rho_{0} / \sigma\right]^{2 /(8-3 \mu)}$

which corresponds to the critical deposition power:

$P_{\mathrm{cr}}=4 \sigma_{B} T_{\mathrm{cr}}^{4} /\left(\rho_{0} r_{0}\right)$

here, $\rho_{0}$ is in unit of $\mathrm{g} / \mathrm{cm}^{3}$ and $r_{0}$ is in unit of $\mathrm{cm}$. There are two regimes: (1) the hydrodynamic regime for $T \ll T_{\mathrm{cr}}$, in which the deposited energy just causes hydrodynamic expansion of the converter, and (2) the radiative regime for $T \gg T_{\text {cr }}$, in which most of the energy is radiated and hydrodynamic expansion is small. The efficiency of converting beam energy $E_{\mathrm{b}}$ into X-rays is given by:

$\eta_{\text {con }}=\frac{E_{\text {rad }}}{E_{\mathrm{b}}}$

By using Eqs. 4 and 5, one can rewrite Eq. 10 in form of:

$\eta_{c o n}=\frac{2 \sigma T^{4}}{P \rho_{0} r_{0}}\left(1-\frac{t_{h}}{t_{b}}\right)$

and by using Eqs. 6 and 11, we will have: 
$\eta_{\mathrm{con}}=1-\frac{(2 \gamma-1) e(T) M_{\mathrm{con}}}{E_{\mathrm{b}}}$

In the radiative regime, one has $T / T_{\mathrm{cr}} \approx\left(2 P / P_{\mathrm{cr}}\right)^{1 / 4}$, and by using:

$\frac{t_{\mathrm{h}}}{t_{\mathrm{b}}}=\frac{e(T) c_{\mathrm{s}}}{\operatorname{Pr}_{0}}$

It is obtained:

$\frac{P}{2 P_{0}}=(\gamma-1)^{\frac{12}{8-3 \mu}}\left[\frac{\tilde{\eta}^{3 \mu}}{(1-\tilde{\eta})^{8}}\right]^{1 / 8-3 \mu}$

where:

$$
\begin{aligned}
\tilde{\eta} & \approx 1-(\gamma-1)^{3 / 2}\left(2 \frac{P_{0}}{P}\right)^{1-3 \mu / 8} \\
& =1-\left(\frac{P_{c r}}{2 P_{0}}\right)^{1-3 \mu / 8}
\end{aligned}
$$

Combining Eqs. 11 to 15, conversion efficiency is derived as a function of the beam and converter parameters $(11,14,15)$, as follows: $\eta_{\text {con }}=1-\frac{(\gamma-1 / 2)}{(\gamma-1)}\left(\frac{P_{c r}}{2 P}\right)^{1-3 \mu / 8}$

\section{Results and discussion}

Figure 2 shows the transport of $10 \mathrm{GeV} \mathrm{Bi}^{+}$ion inside the solid $\mathrm{Be}$ and $\mathrm{Au}$. The beam transport is simulated by SRIM code. The beam starts digging into the material along the axis as the initially heated material rarefies. When the chosen pulse duration is always shorter than the disintegration time, the digging effect plays a minor role. This seems to be a reasonable choice in the context of ICF, since the converter elements for indirect drive need to be well localized to keep the radiation cavity small [14]. Bragg peak is illustrated in Fig. 1b, d. As it is seen low- $Z$ material has higher ion-stopping power and hence has less total internal energy than does high- $Z$ material at a given temperature since the ion beam deposits more energy in a low- $Z$ material. This is shown in Fig. 3.

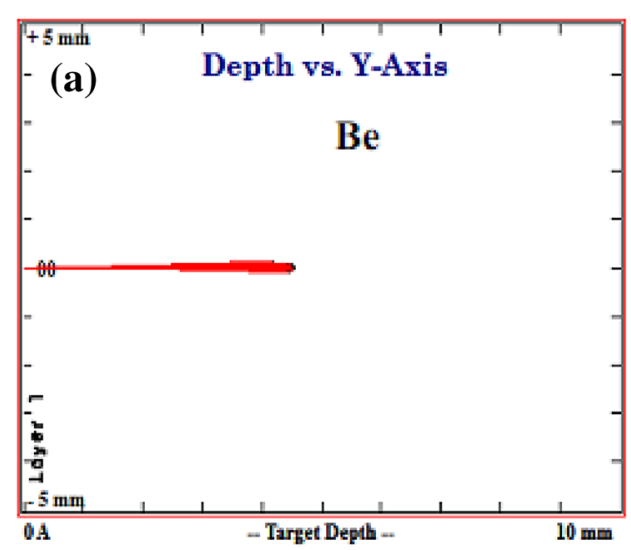

(b)

(Be)
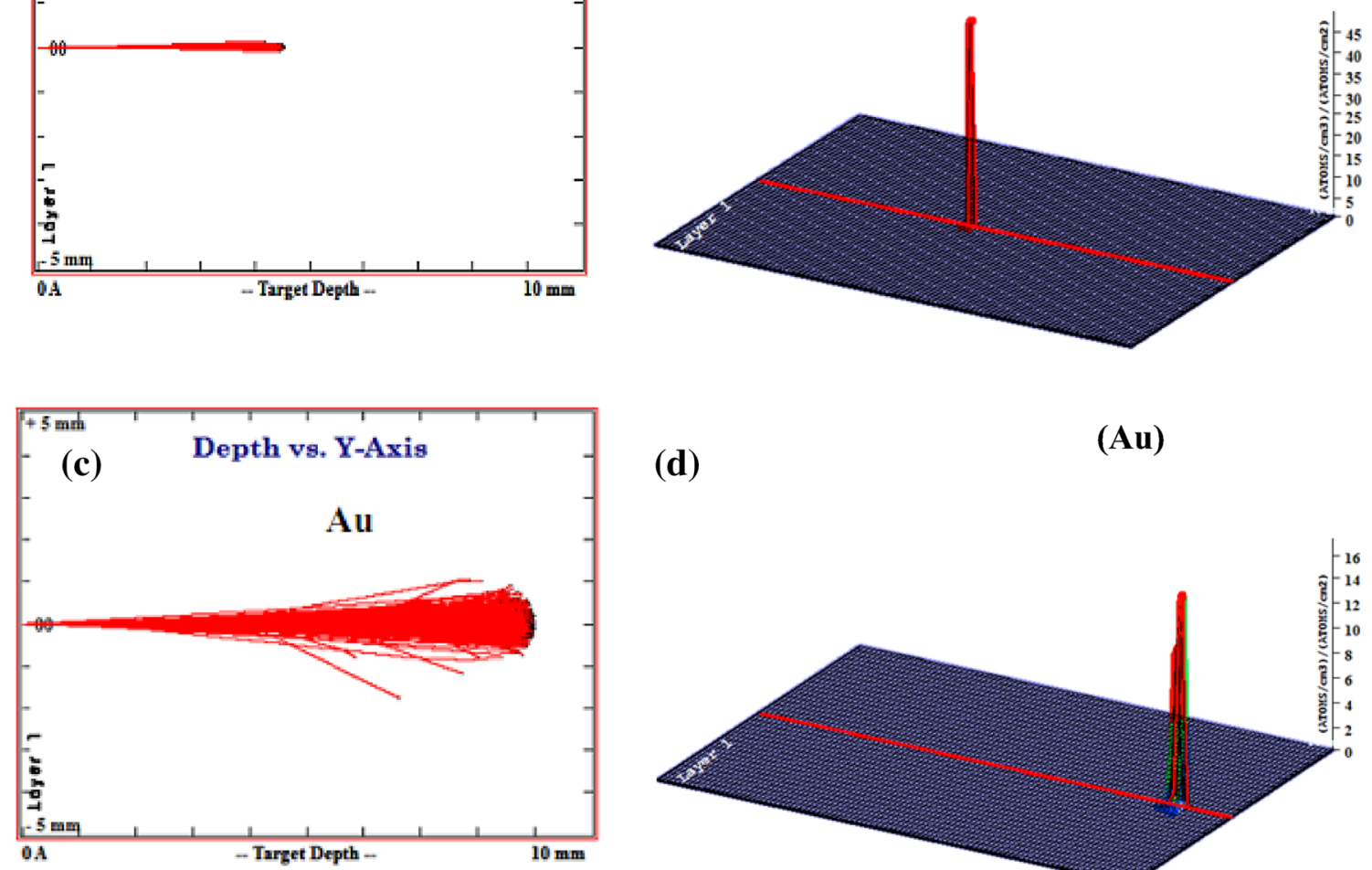

(d)

(Au)

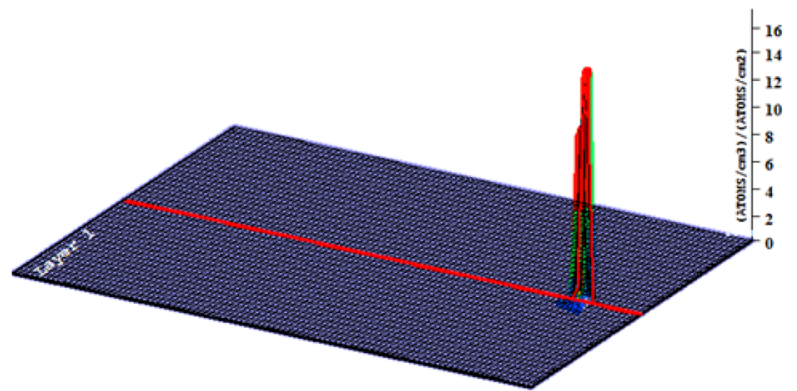

Fig. 2 a, c Simulation of $10 \mathrm{GeV} \mathrm{Bi}{ }^{+}$ion trajectories into solid $\mathrm{Be}$ and $\mathrm{Au}$ at $0^{\circ}$ incidence angle with parameter target depth $=0.1 \mathrm{~cm}$, $\rho_{0}=0.3 \mathrm{~g} / \mathrm{cm}^{3}$. b, d The energy deposition from the ion beam as it penetrates in Be and Au 


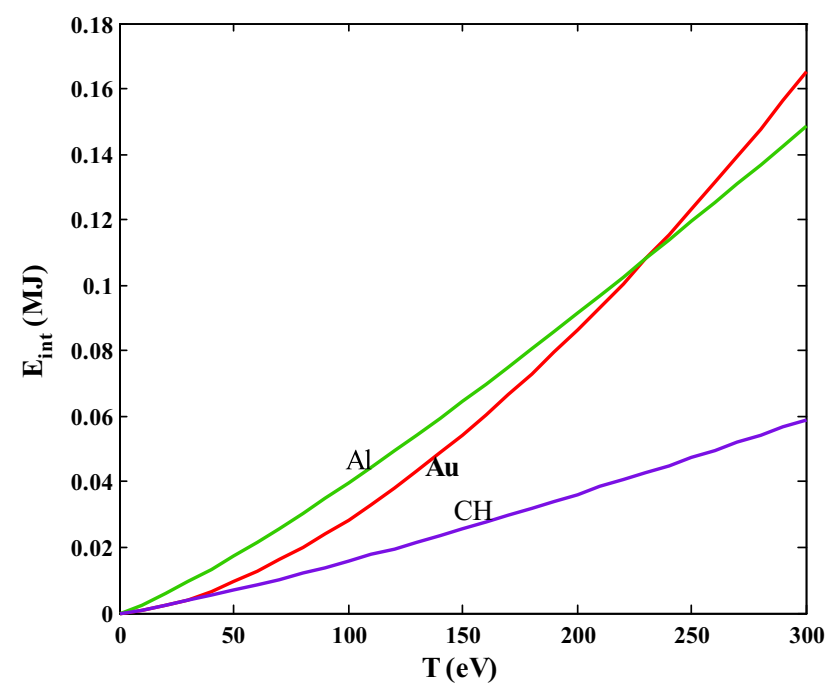

Fig. 3 Internal energy $E_{\text {int }}$ as a function of temperature $T$ for a stretched cylindrical converter with parameters: $r_{0}=1 \mathrm{~mm}$, $\rho_{0}=0.3 \mathrm{~g} / \mathrm{cm}^{3}$

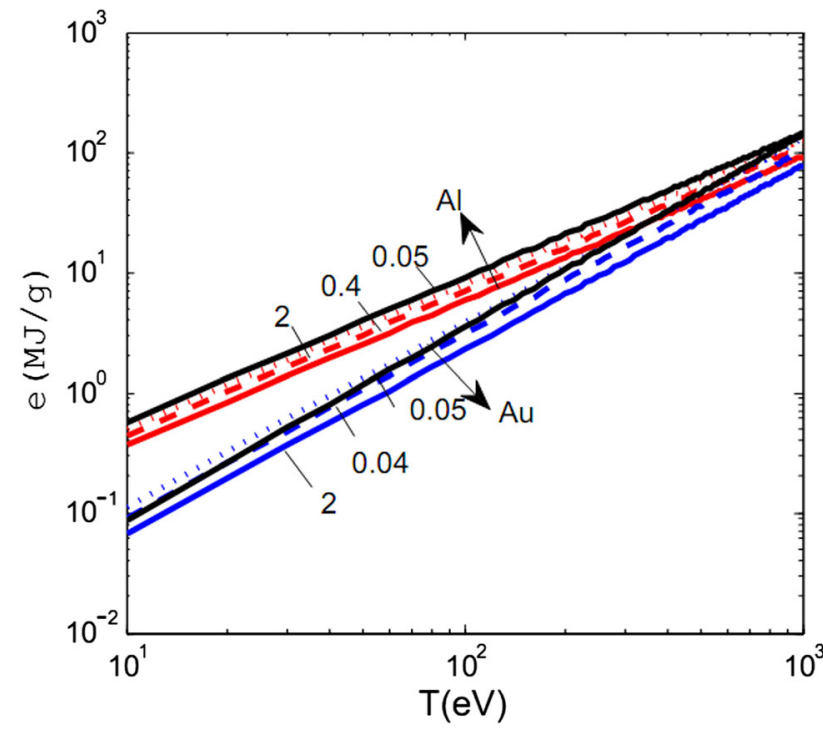

Fig. 4 Gold- and aluminium-specific internal energy $e(\rho, T)=$ $e(T) \rho^{-v}$ versus temperature for different densities. The black lines correspond to the power law relation

The specific internal energy for different densities versus temperature is plotted in Fig. 4. The specific internal energy is an increasing function of the temperature. For a given density, gold has lower specific internal energy than that of aluminium. From Fig. 1, one finds the specific internal energy is about $20 \mathrm{MJ} / \mathrm{g}$ for $300 \mathrm{eV}$ gold, and even more for aluminium and other low- $Z$ materials at this temperature. This puts a severe limit on the usable converter mass. In addition, it is seen that increase of density causes an increase in the specific internal energy.
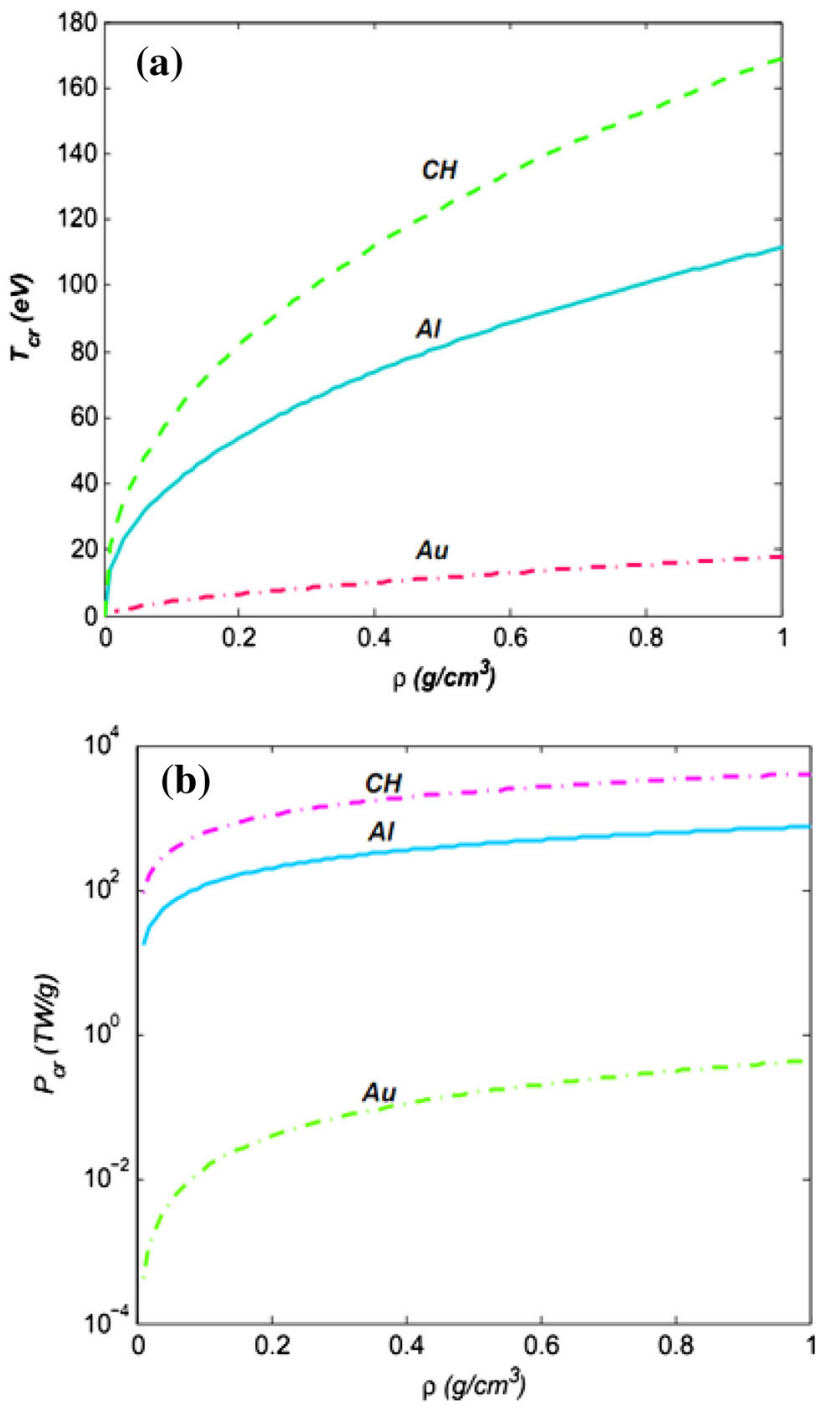

Fig. 5 a The corresponding critical temperature as a function of density for different materials. b The critical deposition power as a function of density for different materials

The critical temperature as a function of density for various materials is plotted in Fig. 5a. It is seen that the critical temperature depends on density and material of a converter. For a given density, gold has a very low critical temperature as compared with aluminium or plastic. It can be observed that with increasing density, critical temperature also increases. The density changes have a significant effect on the low- $Z$ material, for example, when density increases from 0 to 1 , gold represents a temperature difference of about $20 \mathrm{eV}$, while plastic represents a temperature difference about $160 \mathrm{eV}$; high- $Z$ materials such as gold flow into their radiation emission (radiation regime), earlier than low- $Z$ materials. Figure $5 \mathrm{~b}$ shows the critical deposition power as a function of density for different materials. The most 


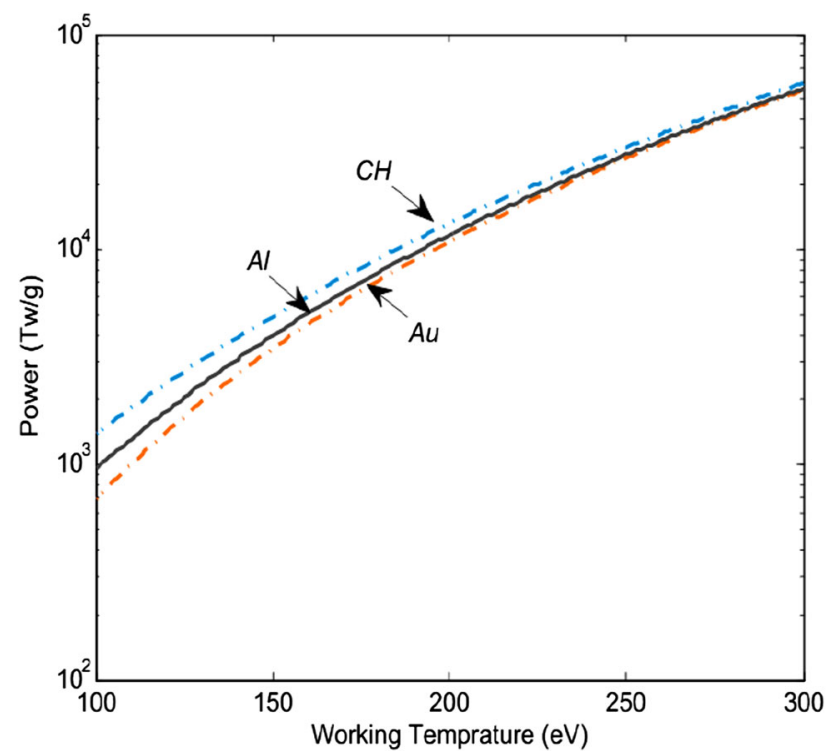

Fig. 6 The converter temperature as a function of specific deposition power $P$. Converter parameters: $r_{0}=0.1 \mathrm{~cm}, \rho_{0}=0.3 \mathrm{~g} / \mathrm{cm}^{3}$

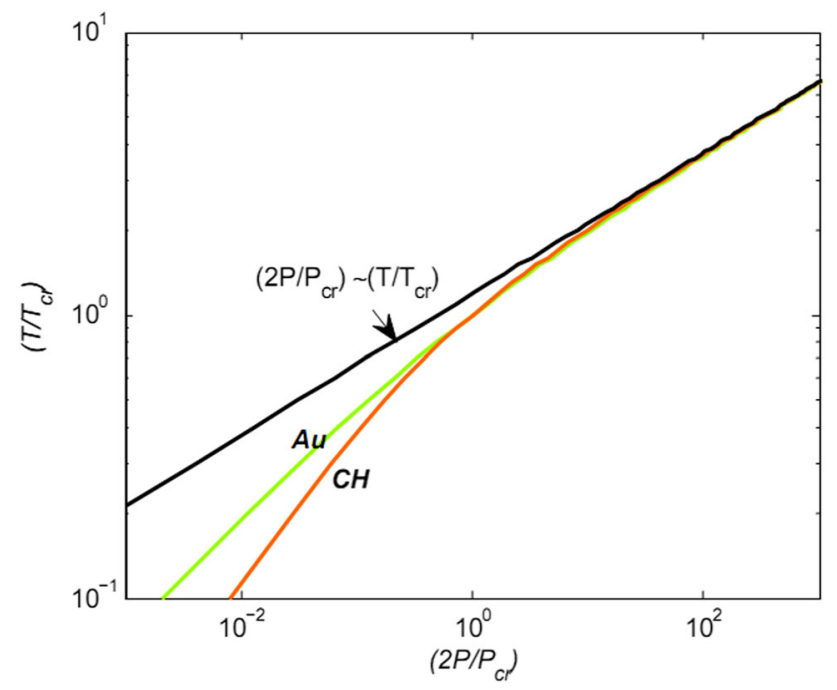

Fig. 7 The converter temperature as a function of specific deposition power $P$

significant point concerning this figure is that critical deposition power for gold is almost one order of magnitude smaller than for aluminium and plastic. This indicates that gold and comparable high- $Z$ elements are superior converter materials. The converter temperature according to the specific deposition power is plotted in Fig. 6. It can be seen that with increasing the power, converter temperature increases. For a given input power, the temperature of $\mathrm{Au}$ is more than that of $\mathrm{Al}$ and $\mathrm{CH}$. The physical reason is that high- $\mathrm{Z}$ materials have higher

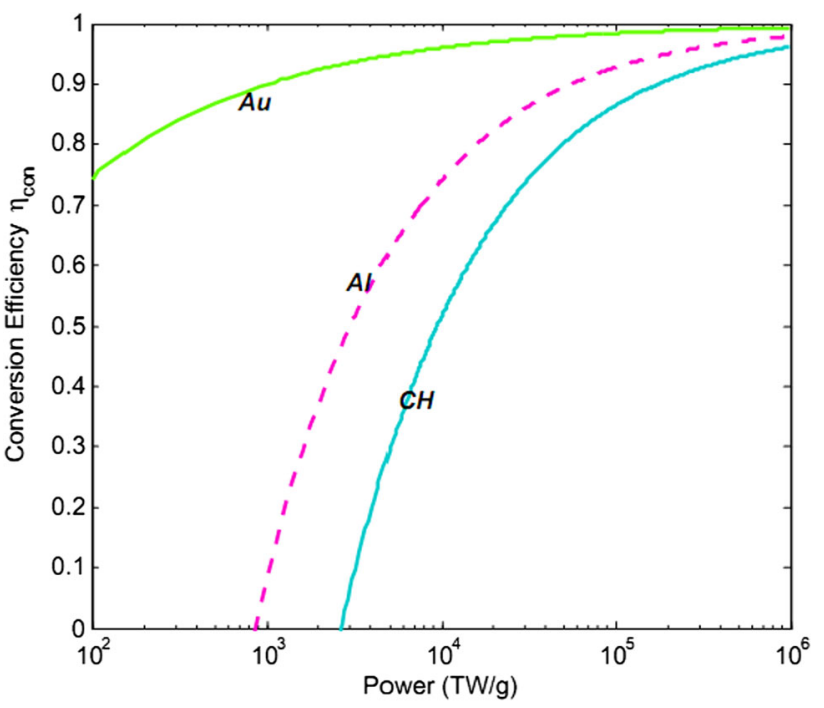

Fig. 8 Conversion efficiency for different materials with parameters: $r_{0}=0.1 \mathrm{~cm}, \rho_{0}=0.3 \mathrm{~g} / \mathrm{cm}^{3}$

temperature at a given internal energy than low- $\mathrm{Z}$ materials. At the power level of $10^{16} \mathrm{~W} / \mathrm{g}$, differences between high- $Z$ and low- $Z$ converter material become less important. The full dependence of temperature on deposition power is plotted in Fig. 7 for gold and aluminium; it can be observed that the relation $\frac{T}{T_{c r}} \approx\left(\frac{2 P}{P_{c r}}\right)^{1 / 4}$ is well fulfilled for $P / P_{0}>1$, in the case of gold even for $P /$ $P_{0}>0.01$. The conversion efficiency according to the specific deposition power for different materials is plotted in Fig. 8. It is found that the conversion efficiency is an increasing function of the specific power deposited by the ion beam. In addition, significantly $\eta_{\text {con }}$ depends on atomic number $\mathrm{Z}$, and conversion efficiency of high- $\mathrm{Z}$ material is more than that of low- $Z$ material. At power level less than $10^{16} \mathrm{TW} / \mathrm{g}$, high- $Z$ material has high conversion efficiency, and for power greater than $10^{16} \mathrm{TW} / \mathrm{g}$, differences between conversion efficiency of high- $\mathrm{Z}$ and low-Z material become less.

\section{Conclusions}

We used a theoretical analysis to achieve an optimal X-ray flux from irradiated converter by heavy ion beam. It is found that converters made of high- $\mathrm{Z}$ material have suitable properties, since they reach higher temperatures at a given specific internal energy. High- $Z$ materials have lower specific internal energy than do low- $Z$ materials. At the power level of $10^{16} \mathrm{~W} / \mathrm{g}$, high conversion efficiency of $70 \%$ or more is possible. At this power level, differences between high- $\mathrm{Z}$ and low- $\mathrm{Z}$ converter material become less important. 
Open Access This article is distributed under the terms of the Creative Commons Attribution 4.0 International License (http://crea tivecommons.org/licenses/by/4.0/), which permits unrestricted use, distribution, and reproduction in any medium, provided you give appropriate credit to the original author(s) and the source, provide a link to the Creative Commons license, and indicate if changes were made.

\section{References}

1. Tahir, N.A., Spiller, P., Shutov, A., Lomonosov, I.V., Piriz, A.R., Redmer, R., Hoffmann, D.H.H., Fortov, V.E., Deutsch, C., Bock, M.: Proposed High Energy Density Physics Research Using Intense Particle Beams at FAIR: The HEDgeHOB Collaboration, IEEE Trans. Plasma Sci. 37, 1267 (2009)

2. Hoffmann, D.H.H., Blazevic, A., Ni, P., Rosmej, O., Roth, M., Tahir, N.A., Tauschwitz, A., Udrea, S., Varentsov, D., Weyrich, K., Maron, Y.: Present and future perspectives for high energy density physics with intense heavy ion and laser beams, Laser Part. Beams 23, 47 (2005)

3. Barnard, J.J., Armijo, J., Bailey, D.S., Friedman, A., Bieniosek, F.M., Henestroza, E., Kaganovich, I., Leung, P.T., Logan, B.G., Marinak, M.M., More, R.M., Ng, S.F., Penn, G.E., Perkins, L.J., Veitzer, S., Wurtele, J.S., Yu, S.S., Zylstra, A.B.: Ion beam heated target simulations for warm dense matter physics and inertial fusion energy, Nucl. Instr. Methods A 606, 134 (2009)

4. Kawata, S., Miyazawa, K., Kikuchi, T., Someya, T.: Directindirect mixed implosion mode in heavy ion inertial fusio, Nucl. Instr. Methods A 577, 332 (2009)
5. Tahir, N.A., Hoffmannt, D.H.H., Maruhnt, J.A., Deutschs, C.:Analysis of Compression, Thermonuclear Burn, and Hydrodynamic Stability of a Reactor-Size Radiation Driven Inertial Fusion Target, Plasma Phys. Cantml. Fusion 37, 447 (1995)

6. Meyer-ter-vehn, J.: Inertial confinement fusion driven by heavy ion beams, Plasma Phys. Control. Fusion 31, 1613 (1989)

7. Basko, M., Lutz, K.J., Maruhn, J.: On possible target design for a heavy ion ignition facility. Nucl. Instr. Methods A 415, 85 (1998)

8. Ramis, R., Ramírez, J., Honrubia, J.J., Meyer-ter-Vehn, J., Piriz, A.R., Sanz, J., Ibáñez, L.F., Sánchez, M.M., de laTorre, M.: A 3 MJ optimized hohlraum target for heavy ion inertial confinement fusion, Nucl. Instr. Methods A 415, 93 (1998)

9. Atzeni, S., Meyer-ter-vehn, J.: The Physics of Inertial Fusion. Clarendon Press, Oxford (2004)

10. Atzeni, S.: Study of Thermal Radiation Generation from Ion Beam Heated Converters, Part. Accel. 37-38, 495 (1992)

11. Pfalzner, S.: An Introduction of Inertial Confinement Fusion. Taylor \& Francis Group, Boca Raton (2006)

12. Duderstad, J.J., Moses, G.A.: Inertial Confinement Fusion. Wiley (1982)

13. Ho, D.D.-M., Lindl, J.D., Tabak, M.: Radiation converter physics and a method for obtaining the upper limit for gain in heavy ion fusion, Nucl. Fusion 34, 1081 (1994)

14. Murakami, M., Meyer-ter-Vehn, J., Ramis, R.: Thermal x-ray emission from ion-beam-heated matter, J. X-Ray Sci. Technol 2 , 127 (1990) 